Case

Report

\title{
Aortic Valve Replacement for Aortic Regurgitation with Rare Left Ventricular Non-Compaction
}

\author{
Hideyuki Tanaka, $\mathrm{MD},{ }^{1}$ Tatsunori Kimura, $\mathrm{MD}, \mathrm{PhD},{ }^{1}$ and Shinji Miyamoto, $\mathrm{MD}, \mathrm{PhD}^{2}$
}

\begin{abstract}
Left ventricular noncompaction cardiomyopathy is a rare type of congenital cardiomyopathy characterized by prematurely arrested compaction of the endocardial and myocardial fibers and the progressive deterioration of left ventricular contractility. This entity is a genetically heterogeneous disorder and has a wide spectrum of presentation from no symptoms to critical disabling congestive heart failure, which can appear at any age. The prognosis is therefore varied. An elderly patient with left ventricular noncompaction underwent aortic valve replacement for associated aortic regurgitation. Follow-up at two years after surgery revealed an improved clinical condition and recovered cardiac function. This is the fourth known aortic valve replacement in a patient with left ventricular noncompaction.
\end{abstract}

Keywords: cardiomyopathy, ventricular noncompaction, aortic valve replacement

\section{Introduction}

Left ventricular noncompaction cardiomyopathy (LVNC) is believed to be a morphogenetic abnormality involving arrested compaction of the loose myocardial meshwork during early embryogenesis. ${ }^{1)}$ The World Health Organization categorizes LVNC as an unclassified cardiomyopathy. ${ }^{2)}$ The pathophysiology of this abnormality is the progressive deterioration of LV systolic function, ${ }^{1)}$ but the cause remains controversial. A relationship between coronary microcirculatory dysfunction and subendocardial ischemia is suspected. ${ }^{3,4)}$ The triad of heart failure, systemic embolism and arrhythmias is the major clinical presentation when the LV systolic function deteriorates. ${ }^{1,5)}$ Various non-invasive imaging modalities have been

${ }^{1}$ Department of Cardiovascular Surgery, Shinbeppu Hospital, Beppu, Oita, Japan

${ }^{2}$ Department of Cardiovascular Surgery, Faculty of Medicine Graduate School of Medicine, Oita University, Yufu, Oita, Japan

Received: February 21, 2012; Accepted: June 7, 2012

Corresponding author: Hideyuki Tanaka, MD. Department of Cardiovascular Surgery, National Hospital Organization Beppu Medical Center, 1473 Uchikamado, Beppu, Oita 874-0011, Japan Email: tanakah@oita-u.ac.jp

(C)2012 The Editorial Committee of Annals of Thoracic and Cardiovascular Surgery. All rights reserved. used to diagnose LVNC including echocardiography, magnetic resonance imaging and computed tomography. Left ventricular noncompaction was initially considered as one cause of critical congestive heart failure in a pediatric population, ${ }^{1)}$ but this abnormality has been identified in an adult population due to advances in imaging techniques. ${ }^{6}$ Cardiac surgery for adult patients with LVNC has rarely been reported. Here we describe aortic valve replacement to treat aortic regurgitation (AR) in a patient with LVNC.

\section{Case Report}

A 74-year-old woman without a family history of cardiovascular disease or sudden death was referred to our hospital with dyspnea that had persisted for three months. The physical findings were unremarkable except for a diastolic murmur at the left sternal edge. A chest $\mathrm{x}$-ray showed cardiomegaly with a cardiothoracic ratio of $70 \%$. Plasma levels of brain natriuretic peptide (BNP) and noradrenalin were elevated to 1540 and $481 \mathrm{pg} / \mathrm{ml}$, respectively. Transthoracic echocardiography revealed extreme regurgitation of the tricuspid aortic valve and diffuse left ventricular (LV) hypokinesis that was particularly severe in the apex, lateral and posterior walls at the mid-ventricular and basal level. The aortic annulus was $23 \mathrm{~mm}$ in 

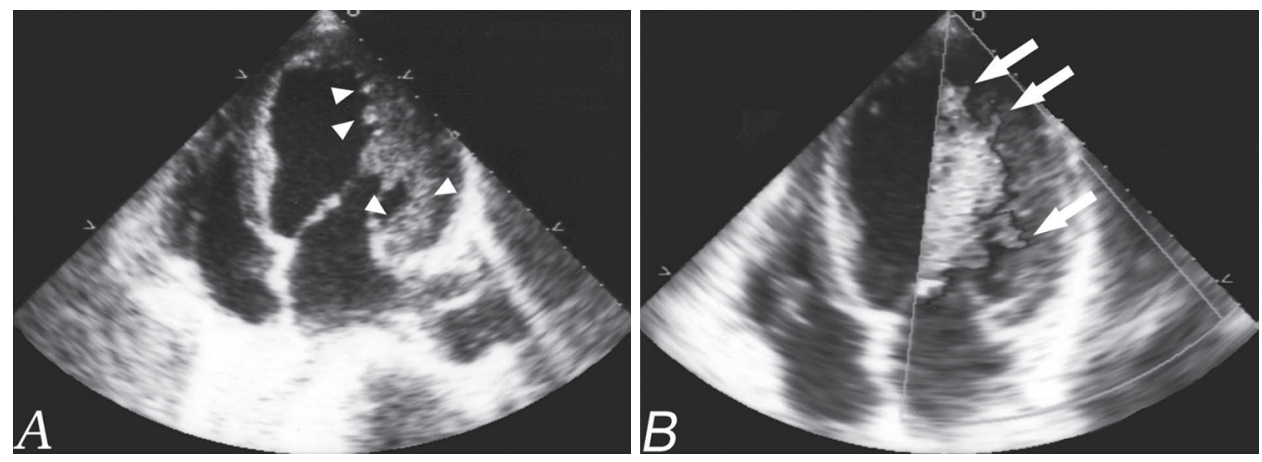

Fig. 1 Two-dimensional echocardiographic appearance of the left ventricle on apical 3-chamber view. (A) noncompacted area along the posterior and lateral wall showing meshwork of trabeculations and intratrabecular recesses (arrowheads), (B) contrast echocardiography showing the direct perfusion of intratrabecular recesses (arrow).

diameter on apical longitudinal axis view. The hypokinetic wall was a bilayered myocardial structure with a thin compacted epicardial layer and a thicker, noncompacted endocardial layer comprising hypertrabeculations and deep intertrabecular recesses. Contrast echocardiography showed a direct passage between the LV cavity and the deep intertrabecular recesses (Fig. 1). The ratio of non-compacted to compacted thickness was about 2:3, which met the diagnostic criteria for LVNC. The left ventricular end-diastolic diameter and ejection fraction (LVEF) were $53.6 \mathrm{~mm}$ and $42.2 \%$, respectively. No other cardiac anomaly was evident. Cardiac catheterization showed Sellers grade $3 \mathrm{AR}$ and LV dilatation with an end-diastolic volume of $233 \mathrm{~mL}$ (volume index of $226 \mathrm{~mL} / \mathrm{m}^{2}$ ). No morphological abnormality of sinus of Valsalva or sinotubular junction was detected (Fig. 2). No significant coronary artery disease was found. The diagnosis was severe AR and LVNC, presenting with New York Heart Association (NYHA) functional class 3 chronic heart failure. She was treated conventionally with medication for heart failure, including an angiotensin-converting enzyme inhibitor and a diuretic and aortic valve replacement was scheduled.

The chest was opened through a median sternotomy and a cardiopulmonary bypass was established by aortic cannulation into the ascending aorta and venous cannulation via the right atrium. Cardiac arrest was established with selective antegrade cold crystalloid. Prominent trabeculations in the left ventricular cavity were observed through the left ventricular outflow tract. The aortic valve had slightly thickened cusps and this was replaced with a 23-mm CarpentierEdwards PERIMOUNT Magna (Edwards Lifesciences LLC, Irvine, CA, USA) in the supra-annular position.
Aortic regurgitation had arisen due to dilatation of the aortic annulus. The patient's postoperative course was uneventful, and she was discharged on postoperative day 27 under medication with an oral anticoagulant, heart failure medication and a beta blocking agent.

Echocardiography one year later showed improved left ventricular function with a LV end-diastolic diameter of $48.1 \mathrm{~mm}$ and a LVEF of $54.8 \%$. The patient remained free of cardiac events at two years, when the symptoms of heart failure improved to NYHA functional class 1. Echocardiography at that point showed the persistent recovery of left ventricular function with a LV end-diastolic diameter of $37.7 \mathrm{~mm}$ and a LVEF of $64.9 \%$. The characteristic features of non-compaction remained unchanged.

\section{Discussion}

Left ventricular non-compaction is a rare congenital cardiomyopathy with a genetic origin that is characterized by numerous, excessively prominent trabeculations and deep intratrabecular recesses. ${ }^{1)}$ This cardiomyopathy is genetically heterogeneous and can be inherited as an autosomal dominant or X-linked recessive disorder. ${ }^{7,8)}$ Echocardiography is considered the reference standard for a diagnosis of LVNC. Multiple trabeculations and deep recesses communicate with the ventricular cavity and the ratio of non-compacted to compacted myocardial thickness is above 2.5,6) Apical and mid-ventricular segments of both inferior and lateral walls are commonly affected. The prevalence of LVNC ranges from $0.05 \%$ to $0.24 \%$ per year in echocardiographic databases. ${ }^{6}$ In pediatric patients, 


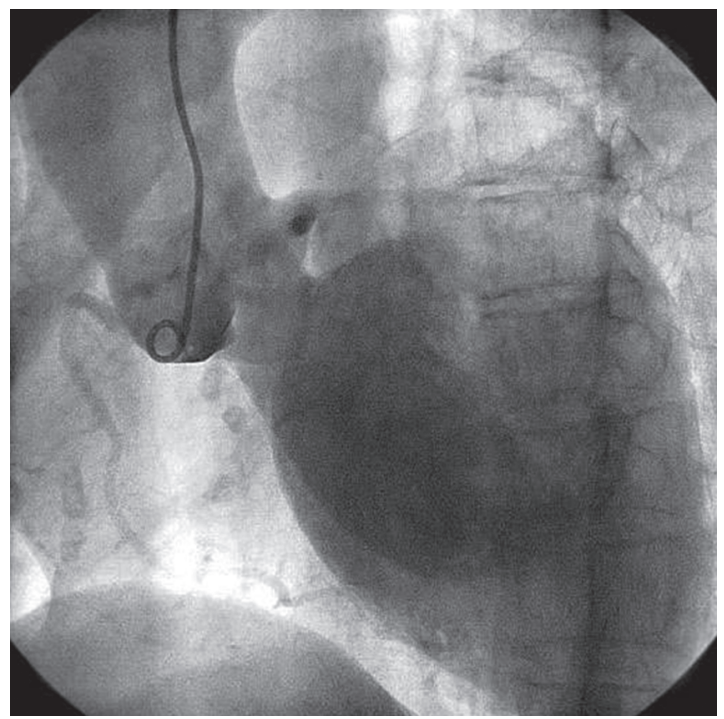

Fig. 2 Aortography revealed Sellers grade 3 AR. The morphology of sinus of Valsalva and sinotubular junction was normal.

LVNC often coexists with other cardiac and extracardiac anomalies such as facial dysmorphism, and the mortality rate is high due to disabling heart failure or sudden death. ${ }^{1}$ On the other hand, LVNC is a distinct entity in adults that has a wide spectrum of presentation from asymptomatic to symptomatic. $\left.{ }^{9}\right)$ The prognosis is therefore varied and clearly better for asymptomatic, than for symptomatic patients. ${ }^{6}$ A larger LV end-diastolic diameter at the time of initial presentation, NYHA functional class $3 / 4$ and chronic atrial fibrillation are significantly more frequent clinical characteristics of non-survivors than in longterm survivors of LVNC. ${ }^{5)}$ However, asymptomatic and symptomatic non-compaction remain morphologically indistinct. Gene analysis for the pathophysiology of LVNC has been recently performed. Shan et al. speculate that genes encoding ion channels are involved in the pathophysiology of LVNC, demonstrating higher prevalence of human cardiac sodium channel alpha-subunit gene (SCN5A) variants in patients with heart failure than in those without. ${ }^{10)}$ They suggest the presence of SCN5A variants increases the severity of LVNC. ${ }^{10)}$

A cure for LVNC has not yet been established. Heart failure, arrhythmia and oral anticoagulation (to prevent systemic emboli) in patients with LV systolic dysfunction are therefore managed by palliative therapies and heart transplantation is recommended for refractory heart failure. ${ }^{5)}$ Our patient presented with chronic heart failure and mild LV systolic dysfunction. Hence, similar to treatment for other cardiomyopathies, we replaced the aortic valve after introducing medication to treat the heart failure. The postoperative course was uneventful. Although intraoperative air trapping from the LV cavity required careful monitoring because of very deep recesses, standard valve replacement was feasible.

To our knowledge, only three case reports have described aortic valve replacement for aortic valve diseases in patients with LVNC. ${ }^{11-13)}$ The associated diseases comprised aortic stenosis in one and aortic regurgitation in two due to a congenital bicuspid valve. This report is the fourth documented replacement of an aortic valve in a patient with LVNC and associated AR due to dilatation of the aortic annulus. Left ventricular noncompaction often coexists with other cardiovascular anomalies. Coexistence with anomalies involving bicuspid aortic valve, ventricular septal defect, sinus of Valsalva aneurysm and aortic coarctation was reported. ${ }^{14,15)}$

Cardiovascular extracellular matrix (ECM) is implicated in cardiovascular development and adult homeostasis. Therefore, defects in ECM or its modifying enzymes could cause of both congenital and adultonset cardiovascular disorders. Versican is a large chondroitin sulfate proteoglycan of the ECM, which is cleaved by proteases of the ADAMTS (A Disintegrinlike and Metalloprotease domain with ThromboSpondin type 1 motifs) family. ${ }^{16)}$ It was suggested that versican cleavage was required for correct cardiovascular development and adult homeostasis in a recent report in which mice with reduced versican cleavage due to ADAMTS9 haploinsufficiency were analyzed. ${ }^{17)}$ In this report, anomalies in the aortic wall, valvulosinus and valve leaflets were specifically found in adult mice with ADAMTS9 haploinsufficiency, and furthermore, left ventricular noncompaction was also found in mice with that. ${ }^{17)}$ In present case left ventricular noncompaction coexisted with dilatation of the aortic annulus, which may reflect common pathway in origin.

A long-term follow-up of 34 adult patients with LVNC showed that of the $35 \%$ of them died, half was due to sudden death, and $12 \%$ had received a heart transplant. ${ }^{5)}$ The prognosis of patients with LVNC is generally poor when clinically symptomatic or when LV systolic function deteriorates. Our patient presented with heart failure at the time of admission. Regardless, our patient's symptoms had improved within two years and the cardiac function has persistently recovered. Because our patient's clinical presentation appeared after a long asymptomatic course, we presumed that she had LVNC with a favorable prognosis, and that severe 
AR rather than LVNC worsened her clinical condition. Therefore, our patient's clinical condition might have been improved by the aortic valve replacement.

\section{Conclusion}

The present case indicates that cardiac surgery for associated valve disease can improve the clinical condition and recover cardiac function even among patients with left ventricular non-compaction.

\section{Disclosure Statement}

We disclose that we have no conflict of interest.

\section{References}

1) Chin TK, Perloff JK, Williams RG, et al. Isolated noncompaction of left ventricular myocardium. A study of eight cases. Circulation 1990; 82: 507-13.

2) Richardson P, McKenna W, Bristow M, et al. Report of the 1995 world health organization/international society and federation of cardiology task force on the definition and classification of cardiomyopathies. Circulation 1996; 93: 841-2.

3) Junga G, Kneifel S, Von Smekal A, et al. Myocardial ischaemia in children with isolated ventricular noncompaction. Eur Heart J 1999; 20: 910-6.

4) Jenni R, Wyss CA, Oechslin EN, et al. Isolated ventricular noncompaction is associated with coronary microcirculatory dysfunction. J Am Coll Cardiol 2002; 39: 450-4.

5) Oechslin EN, Attenhofer Jost CH, Rojas JR, et al. Long-term follow-up of 34 adults with isolated left ventricular noncompaction: a distinct cardiomyopathy with poor prognosis. J Am Coll Cardiol 2000; 36: 493-500.

6) Stöllberger C, Finsterer J. Left ventricular hypertrabeculations/noncompaction. J Am Soc Echocardiogr 2004; 17: $91-100$.
7) Ichida F, Tsubata S, Bowles KR, et al. Novel gene mutations in patients with left ventricular noncompaction or Barth syndrome. Circulation 2001; 103: 1256-63.

8) Sasse-Klaassen S, Gerull B, Oechslin E, et al. Isolated noncompaction of the left ventricular myocardium in the adult is an autosomal dominant disorder in the majority of patients. Am J Med Genet A 2003; 119A: 162-7.

9) Ritter M, Oechslin E, Sütsch G, et al. Isolated noncompaction of the myocardium in adults. Mayo Clin Proc 1997; 72: 26-31.

10) Shan L, Makita N, Xing Y, et al. SCN5A variants in Japanese patients with left ventricular noncompaction and arrhythmia. Mol Genet Metab 2008; 93: 468-74.

11) Wilbring M, Kappert U, Schön S, et al. Aortic valve replacement in noncompaction cardiomyopathy at twoyear follow-up. J Card Surg 2009; 24: 684-6.

12) Ohki S, Moriyama Y, Mohara J, et al. Aortic valve replacement for aortic regurgitation in a patient with left ventricular noncompaction. Ann Thorac Surg 2009; 87: 290-2.

13) Wrigley BJ, Rosin M, Banerjee P. Replacement of a congenital bicuspid aortic valve in a patient with left ventricular noncompaction. Tex Heart Inst J 2009; 36: 241-3.

14) Myers PO, Tissot C, Cikirikcioglu M, et al. Complex aortic coarctation, regurgitant bicuspid aortic valve with VSD and ventricular non-compaction: a challenging combination. Thorac Cardiovasc Surg 2011; 59: 313-6.

15) Unlu M, Ozeke O, Kara M, et al. Ruptured sinus of Valsalva aneurysm associated with noncompaction of the ventricular myocardium. Eur J Echocardiogr 2008; 9: 311-3.

16) Apte SS. A disintegrin-like and metalloprotease (reprolysin-type) with thrombospondin type 1 motif (ADAMTS) superfamily: functions and mechanisms. J Biol Chem 2009; 284: 31493-7.

17) Kern CB, Wessels A, McGarity J, et al. Reduced versican cleavage due to Adamts9 haploinsufficiency is associated with cardiac and aortic anomalies. Matrix Biol 2010; 29: 304-16. 